\title{
PRECISION SOLAR ASTROMETRY FROM SOHO/MDI
}

\author{
J.R. KUHN \\ National Solar Observatory/Sac Peak \\ and Michigan State University \\ P.O. Box 5, Sunspot NM 88349 \\ and Dept. Physics-Astronomy, MSU, E. Lansing, MI 48824, \\ $U S A$ \\ R. BOGART, R. BUSH, L. SÁ AND P. SCHERRER \\ Stanford University, CSSA-HEPL, Stanford CA 94305-4055, \\ USA \\ AND \\ X. SCHEICK \\ Michigan State University, E. Lansing MI, 48824, USA
}

\begin{abstract}
The SoHO/MDI experiment generates a continous record of the solar limb brightness using 1.96" pixels. Because there is no atmospheric blurring, these data allow measurements of solar limb brightness and shape changes with a precision that has not been achieved from the ground. The first results of a 1 month astrometric timeseries from the MDI solar structure program will be described here.
\end{abstract}

\section{Introduction}

The solar limb is, potentially, a sharp spatial fiducial reference with which we can hope to detect the effects of: solar oscillations (both p- and g-modes), the gravitational quadrupole moment (or the solar oblateness), and changes in the solar radius (perhaps as a diagnostic for the solar luminosity cycle). Ground-based attempts to measure a changing radius or limb shape have a long history but, unfortunately, corresponding observations from space are virtually nonexistent.

Hill's group (cf. Brown et al. 1978) pioneered modern astrometric attempts to measure solar oscillations, although their conclusions were never reproduced. Later astrometric measurements from limb photometry failed 
to detect solar g-modes, but did produce interesting upper limits to the possible amplitudes of such modes (cf. Kuhn et al. 1986).

A recent review of the solar oblateness problem (Sofia et al. 1994) reveals that the ground and balloon-based measurements are not consistent. While Dicke et al. (1987) hinted that the sun may be changing its shape with the solar cycle, the recent balloon data do not confirm this. Since atmospheric blurring is orders of magnitude larger than the ultimate accuracy required, these measurements are quite difficult and hours or days of observations (typically) are needed to achieve milliarcsecond accuracy in oblateness measurements from the ground. It seems likely that the apparent differences between many of these experiments are dominated by poorly understood systematic errors.

The solar radius measurements are also in disarray. For example, Ribes et al. (1991) and Parkinson et al. (1980) describe the inconsistency between the many ground-based astrometric measurements of the sun's radius. While there are hints of periodic solar radius variations over timescales of 1000 days to 80 years, the measurements are generally neither consistent, nor conclusive. The evidence for long term secular variations is similarly inconclusive.

The solar limb position varies at physically interesing angular scales of milliarcseconds (the solar shape) and microarcseconds (due to oscillations). Since atmospheric "angle of arrival" fluctuations due to turbulence occur at a scale of an arcsecond, the advantage of space-based astrometric observations is obvious. Until SoHO, and except for a relative few continuum solar images from the Yohkoh experiment, there have been no space experiments that could provide interesting full-disk solar astrometric data.

\section{Data Analysis}

The MDI experiment (Scherrer et al. 1995) was primarily designed to obtain full-disk and higher resolution doppler "images" of the sun for helioseismic analysis. In full-disk mode, images are formed on a $1024 \times 1024$ pixel CCD at a scale of about 1.96 arcseconds/pixel. Doppler and magnetic information is computed from five $94 \mathrm{~mA}$ passbands, spaced $75 \mathrm{~mA}$ apart near the 6768 A Ni I line. A continuum image is computed from an algebraic combination of the five filtergrams in an expression that minimizes the "leakage" of the doppler signal (Scherrer et al. 1995). The ray path through the instrument traverses literally dozens of optical elements so that flat-field calibration is essential. Spatially displaced images were obtained early in the mission to allow flat-fielding by using the Kuhn et al. (1991) technique. Slow drifts in the optical elements produce a temporally increasing, but low spatial frequency flat-fielding error. The differential pixel-to-pixel intensity cali- 
bration is estimated to be accurate to about $1 \%$.

Without atmospheric blurring the dominant error in the limb position measurements result from optical aberrations in the instrument and uncalibrated gain variations in the instrument-detector system. The latter effect is minimized by applying a flat-fielding technique that derives the calibration from the data to be calibrated. The former error is minimized because the spacecraft is in a relatively stable environment and most of the optical aberrations are stable, at least over the timescales in which we observe a temporally variable solar limb position. We'll discuss the magnitude of the fixed instrumental aberration problem below in the context of the oblateness measurements. From a least-squares analysis of the solar limb profile, it can be shown then that the dominant time-variable astrometric error, corresponding to the $1 \%$ flat-fielding uncertainty, is of order 0.01 pixel (about 20 milliarcsec) in the derived limb position. Since there are many independent limb pixels in a single measurement, and a possibility of many independent measurements, the statistical error in the solar shape or the limb position spectrum can be quite small.

Image data from MDI are obtained with a 1 min cadence. Except during prearranged "campaigns" there is insufficient downlink bandwidth to return all of these data to the ground station. An annulus of approximately 6 pixels around the solar limb is temporally averaged with a 24 min Gaussian weighting, and sampled and downloaded every 12 minutes. The data described here were taken after May 1, 1996 during a 34 day period. An 8 hour full-disk intensity dataset was also obtained during a campaign in March. These data were sampled with a 1 min cadence and were reduced to limb observations in the same manner as the $12 \mathrm{~min}$ continuous limb observations.

There are approximately 20,000 pixels from each image near the limb which are assigned to radial and angular bins. The image center in pixel units is computed using a robust iterative least-squares technique (McWilliams and Kuhn 1992) which repeatably finds the solar image center to about 0.01 pix. Each limb intensity measurement is assigned to one of 40 radial, $r_{i}$, and 512 angular, $\theta_{j}$, bins as $I\left(r_{i}, \theta_{j}\right)$. An angular average limb profile $I_{0}(r)$ is also computed for each dataset. A least-squares fit of the data in each angular bin of the form $I\left(r_{i}, \theta_{j}\right) \rightarrow\left(1+\alpha_{j}\right) I_{0}\left(r_{i}-\beta_{j}\right)$ is performed. From this calculation two parameter sets, $\alpha_{j}$ and $\beta_{j}$, describe the brightness deviation and spatial limb shift in each of 512 angular bins around the solar image. From a timeseries of limb data we thus derive twodimensional datasets, $\alpha_{j}\left(t_{k}\right)$ and $\beta_{j}\left(t_{k}\right)$, that describe the limb brightness changes and angular limb shift at times, $t_{k}$. Because of limited space in this report, the following discussion focuses on the angular limb position timeseries. 


\section{Observing Limb p-modes and Searching for g-modes}

We have used 443 observations with a 1 min cadence to "calibrate" our technique by looking for p-modes in the $\beta_{j}\left(t_{k}\right)$ timeseries. For this purpose the 2-dimensional limb displacement power spectrum is computed as $F\left(K_{j}, \omega_{k}\right)=\left\|\sum_{r s} \beta_{r}\left(t_{s}\right) \exp i t_{s} \omega_{k} \exp i K_{j} \theta_{r}\right\|^{2}$.

The photospheric radial velocity of solar acoustic p-modes are most naturally spatially decomposed into spherical harmonics, $Y_{l m}(\theta, \phi)$, where $\theta$ and $\phi$ correspond to heliocentric colatitude and longitude coordinates. It can be shown that for spherical harmonics $l$ greater than about 40 that a given acoustic mode specified by $l m$ projects, primarily, onto "limb harmonic" $K_{j}=l$ and $K_{j}=l+2$. Thus, the ridge structure of the 2-dimensional $k-\omega$ acoustic doppler spectrum should be visible in the $F(K, \omega)$ limb spectrum - which was obtained from fundamentally one-dimensional spatial data. Figure 1 shows a greyscale image of $\log _{10}\left[F\left(K_{j}, \omega_{k}\right)\right]$ where the limb shape power is plotted in units of pixel ${ }^{2}$. For 5 -min period acoustic modes with a velocity amplitude of $15 \mathrm{~cm} / \mathrm{s}$, the shape amplitude will be about $10^{-5}$ arcsec or 5 micropixels (corresponding to a power of about $3 \times 10^{-11}$ in the units of Fig. 1 . The excess power and p-mode ridge structure at $\omega=3 \mathrm{mHz}$ is quite clear and the amplitude is consistent with known 5 -min period radial velocity amplitudes.

The linear properties of gravity modes have been well studied (cf. Berthomieu and Provost 1990) although they have never been conclusively detected. Observational limits on g-mode velocity amplitudes with frequencies less than 100 microhertz are a few $\mathrm{cm} / \mathrm{s}$ (Garcia et al. 1988). Limits on $l=2$ modes from limb astrometry (oblateness) observations are at a similar level (Kuhn et al. 1986). Some speculative calculations (cf. Kumar et al. 1996) suggest that photospheric g-mode velocity amplitudes might only be at the level of $1 \mathrm{~mm} / \mathrm{s}$ or smaller.

Because solar g-modes have small surface velocity amplitudes and low frequencies, there is an advantage to using astrometric techniques, rather than doppler measurements, to search for them. A fixed velocity amplitude results in an increasing shape amplitude for modes of lower frequency. Similar to the p-mode analysis above, we have analysed 34 days of 12-min cadence limb data. The spectral analysis is sensitive to shape oscillations with frequencies between a few and a few hundred microhertz. Figure 2 shows the mean noise power averaged over $l=2-509$. The strong peaks near periods of 1 hour are harmonics of the $96 \mathrm{~min}$ periodic interruption of the data when MDI obtains magnetograms. During this time the temperature of the MDI optics changes slightly in a manner which affects the image at the level of 10 's of micropixels. A "shoulder" in the power spectrum near periods of 10 hours is significant and appears to be caused by the sun. 


\section{Log Beta}

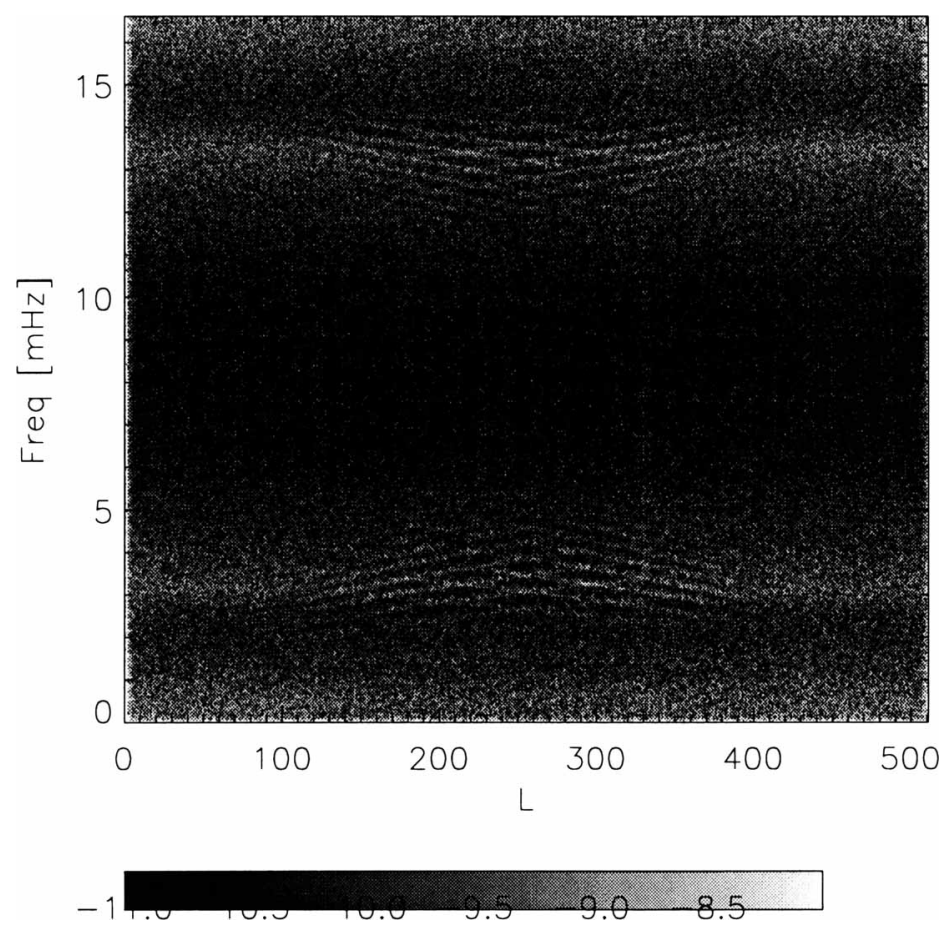

Figure 1. Limb Shape Power Spectrum

Figure 3 shows the averaged shape power for the low $l$ modes expressed in terms of the effective velocity amplitude (in units of $\mathrm{mm} / \mathrm{s}$ ). The shape noise power approaches a few $\mathrm{mm} / \mathrm{s}$ in these data with no outstanding evidence of $g$-mode excitation.

The low frequency limb position data has also been analysed by limb angle to determine the power spectrum of the limb displacement as a function of solar latitude. This analysis sheds some light on the origin of the shoulder in the power spectrum in Fig. 2. We find maxima in the limb position power spectra which vary with latitude. At high latitutes the peak occurs near zero frequency and at the equator the peak occurs at a frequency of about 25 microhertz. The amplitude of the peak corresponds to a limb displacement of about $0.8 \mathrm{~km}$, and the shape of the spectrum (with latitude) suggests a rotating surface distortion pattern (similar to the supergranulation) with a transverse scale at all latitudes of about $6 \times 10^{4} \mathrm{~km}$. This unusual signal clearly requires additional study. 


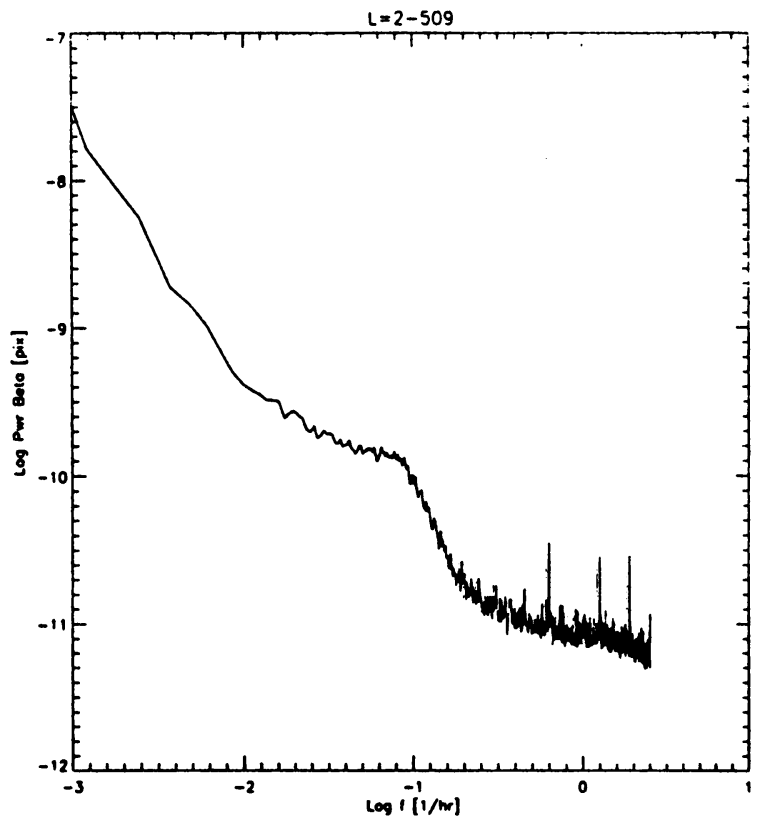

Figure 2. Average Low Frequency Shape Power Spectrum

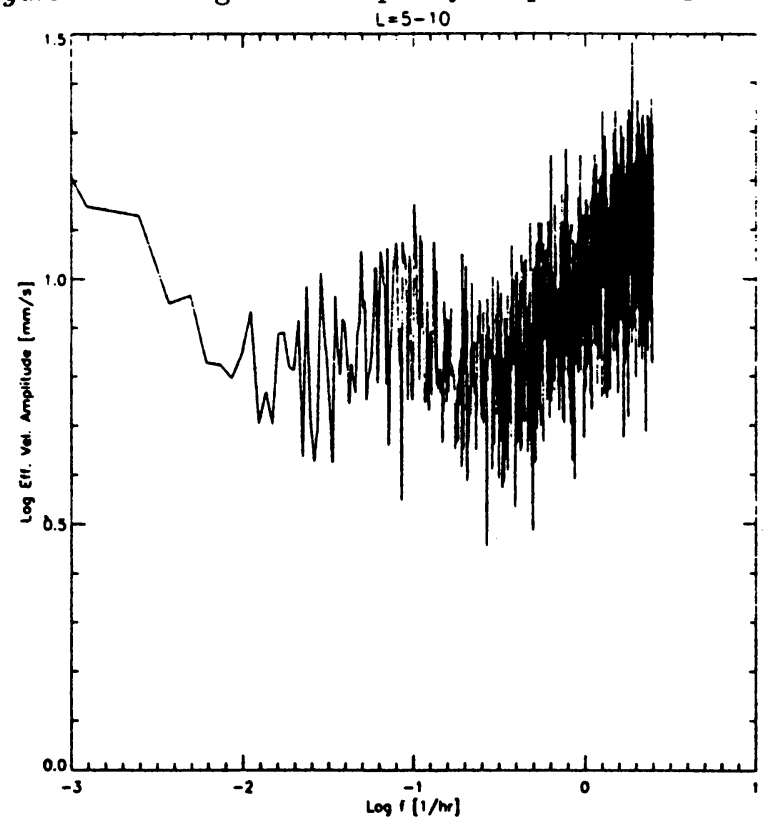

Figure 3. Low $l$ Effective Shape Velocity Noise

\section{The Solar Oblateness and Radius}

By rolling the spacecraft the static solar shape can be extracted from the much larger limb distortion caused by the complex MDI optics. If the limb 


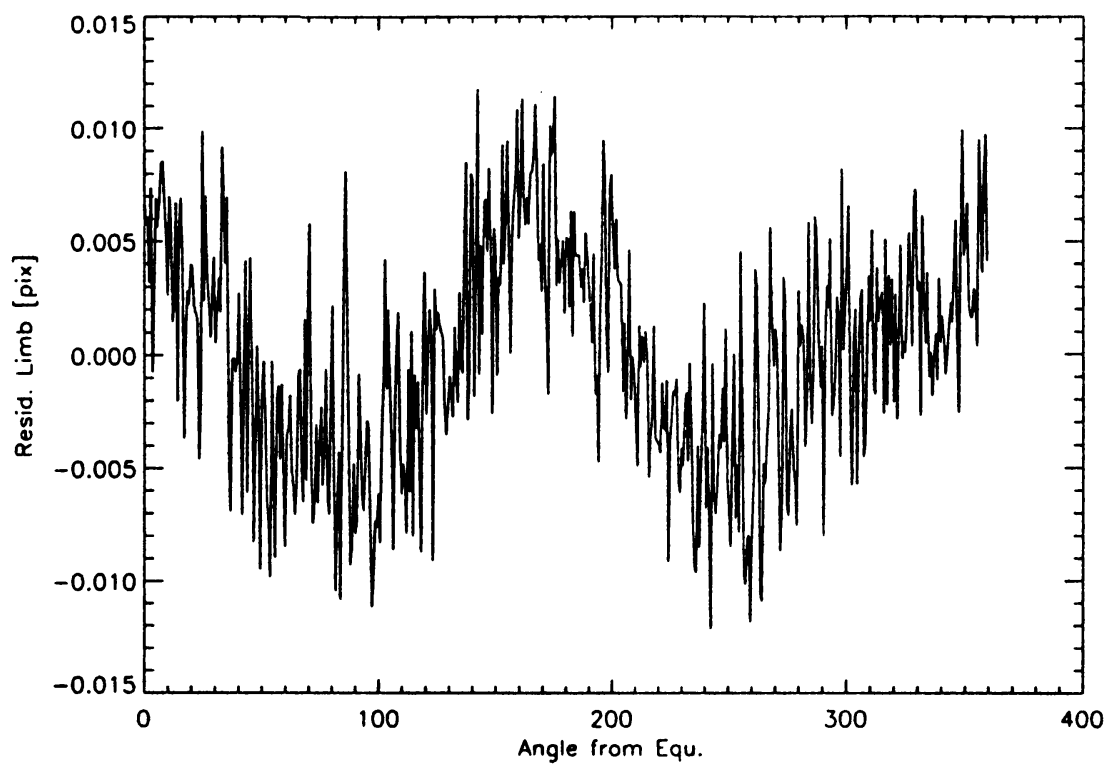

Figure 4. Residual Solar Limb Shape

position as a function of camera-fixed angle $\theta$ (and for a given roll angle $\theta_{i}$ ) is $d_{i}(\theta)=n(\theta)+s\left(\theta+\theta_{i}\right)$ then $d_{i}(\theta)$ is the observed limb shape, $n(\theta)$ is the limb shape due to optical or other instrumental effects, and $s(\theta)$ is the actual solar limb shape. We have analysed only a few minutes of MDI data from roll angles of $0,30,60,90,137,180$, and 270 degrees. From the data taken at angles spaced by 90 degrees the solar and instrumental contributions can be separated. Thus, it can be shown that, $n(\theta)=0.25\left(d_{0}+d_{90}+d_{180}+d_{270}\right)$ is accurate for spatial harmonics through the quadrupole distortion. The intrinsic solar limb shape can then be computed from $d_{i}\left(\theta-\theta_{i}\right)-n\left(\theta-\theta_{i}\right)=$ $s(\theta)$ and averaged over data obtained at all roll angles. Figure 4 shows the solar limb shape, $s(\theta)$, where the solar equator corresponds to the angular origin and the limb position is plotted in pixel units. For comparison, the instrumental contribution to the limb position, $n(\theta)$, has an amplitude of about 0.2 pixels. The function $s(\theta)$ corresponds to a solar oblateness of $\left(r_{e q}-r_{\text {pole }}\right) / r_{e q}=9.6 \pm 0.8 \times 10^{-6}$. An optimum roll sequence and a few more minutes of data should easily decrease the measurement uncertainties here. Nevertheless this measurement is several times more accurate than the ground-based observations, and suggests that the sun's shape does not change significantly with the solar cycle.

The solar radius has also been determined from the mean radial limb profiles from each limb dataset. A cross-correlation analysis of the radial derivative in the limb profiles has proven to be a robust radius estimator. 
These data show "statistical" noise at the level of about 100 micropixels, but there are larger systematic variations which are not completely understood. For example we see an apparent solar radius change of about 0.006 " immediately after MDI obtains magnetograms. These effects, and others, are undoubtably related to changes in the effective focal length of the imaging system, perhaps from small changes in the optics temperature due to the non-constancy of the MDI operating mode.

\section{Prospects, Conclusions and Acknowledgments}

The environment that SoHO now lives in has proven to be remarkably stable and well suited for high precision solar astrometry. While MDI was not specifically optimised for these measurements, and we are only beginning to explore these data, we've seen enough to realize that the astrometric SoHO results will be important.

The MDI experiment is a success because of the efforts of many. At the Lockheed Palo Alto Research Laboratory in Palo Alto CA, W. Rosenberg, L. Springer, T.D. Tarbell, A. Title, C. J. Wolfson and I. Zayer are particularly responsible. Several others (largely responsible for the development of the SOI Science Support Center) have been critical to making this research possible: J. Aloise, L. Bacon, K. Leibrand, V. Johnson, K. Scott, and J. Suryanarayanan. The MDI engineering team consisted of D. Akin, B. Carvalho, R. Chevalier, D. Duncan, C. Edwards, N. Katz, M. Levay, R. Lindgren, D. Mathur, S. Morrison, T. Pope, R. Rehse, and D. Torgerson. Finally, Peter Milford helped with early attempts to measure the solar limb position from Yohkoh data while we were developing the software now used.

\section{References}

Berthomieu, G., Provost, J. (1990) Astron. \& Astrophys., 227, 563.

Brown, T.M., Stebbins, R.T., and Hill, H.A. (1978) Ap. J., 223, 324.

Dicke, R. H. (1981) Proc. Natl. Acad. Sci, 78, 1309.

Dicke, R. H., Kuhn, J.R., and Libbrecht, K.G. (1987) Ap. J., 318, 451.

Garcia, C., Pallé, P.L., and Cortes, T. (1988) Seismology of the Sun and Sun-like Stars (ESA SP-286), ed. E. J. Rolfe (Paris: ESA), 353.

Kuhn, J.R., Lin, H., and Loranz, D. (1991) Publ. Astron. Soc. Pac., 103, 1097.

Kuhn, J.R., Dicke, R.H., Libbrecht, K.G. (1986) Nature, 319, 128.

Kumar, P., Quataert, E., Bahcall, J.N. (1996) Ap.J.,458,83.

McWilliams, T, and Kuhn, J.R. (1992) SOI Technical Note 83 Stanford, CA.

Parkinson, J.H., Morrison, L.V., Stephenson, F.R. (1980) Nature, 288, 548.

Ribes, E., Beardsley, B., Brown, T.M., Delache, Ph., Laclare, F., Kuhn, J.R., Leister, N.V. (1991) The Sun in Time (Sonett, C., Giampapa, M., Matthews, M., eds.) Univ. Ariz. Press, Tucson, 59.

Scherrer, P.H. et al. (1995) Solar Phys., 162, 129.

Sofia, S., Heaps, W., Twigg, L.W. (1994) Ap. J. Suppl., 427, 1048. 